\title{
Evaluation of the behaviour of particulate polymeric coatings in a corrosive environment. Influence of the concentration of metal particles
}

\author{
N. Kouloumbi, G.M. Tsangaris, A. Skordos, S. Kyvelidis \\ National Technical University, Chemical Engineering Dept., Materials Science and Engineering \\ Section, 9 Iroon Polytechniou Street, Athens 157 80, Greece
}

\begin{abstract}
In this work pretreated steel specimens were coated by a spinning process with particulate polymeric composites consisting of an epoxy resin (DOW 33 1) and iron powder. Applied coatings were roughly $70 \mathrm{pm}$ thick and the contained quantity of iron particles was varied (7.5, $15,30 \% \mathrm{wt} . / \mathrm{wt}$.) . The effect of the presence of iron particles in the coatings as well as the influence of their concentration on the evaluation of the coatings' behaviour in a corrosive environment $(3.5 \% \mathrm{NaCl})$ was studied. Electrochemical impedance spectroscopy, corrosion potential, corrosion current density (Tafel) and dielectric measurements were performed. Minor differences in the anticorrosive behaviour of the coatings were observed irrespective of the iron content in the coating. Effective resistance inhibition action of the composite coatings was diminished with the increase of exposure time to the corrosive environment being in all cases very close to that of the pure epoxy resin coatings.
\end{abstract}

Keywords: Particulate polymeric coatings; Corrosion

\section{Introduction}

Epoxy resins, used as binder for industrial coatings, have become increasingly important [1] representing perhaps the best combination of mechanical properties and corrosion resistance [2, 3], thus providing outstanding service under severe conditions [1, 2]. Demands for coatings with superior technical characteristics have induced the use of composite coatings, which usually represent an extremely strong product. Glass, polyaramide or graphite fibres or flakes are employed to offer additional strength and improved abrasion resistance [1, 4, 5]. Glass flake-reinforced coatings are able to combat many of the most severe corrosive 
environments, possessing low permeability for water and corrosive species and low conductivity [6]. Composites with graphite fibres form a durable protective coating, though it is known [7] that when coupled to ordinary metals, in the presence of moisture, they promote galvanic corrosion of any metal less noble than platinum.

Highly conductive particulate polymer composites have recently found numerous important industrial applications [8], e.g. for EM1 shields [8, 9], radar absorbing materials [7], static dissipative plastics [8, 10], heating devices [8] and conductive coatings [11], as they exhibit increased thermal and mechanical properties and contribute to structural, optical and electrical needs $[7,12,13]$.

Data concerning the protective action of particulate polymeric coatings justify the excellent barrier protection that zinc-rich coatings offer [14-16] and demonstrate that composites with metal fillers (Fe, Al, Zn) exhibit similar [17] or slightly lower [18] protective properties than that of pure epoxy coatings.

Conductive fillers can greatly alter conductivity and dielectric characteristics of the polymeric matrix in which they are dispersed [19], affecting significantly the diffusion of

water to the metal-coating interface and consequently the appearance of galvanic interactions. Water in a coating is the necessary factor for the start of the corrosion process [20], which is mainly blistering [21] also resulting in loss of adhesion and deterioration of the mechanical properties [22].

The transport path which determines the conductivity in such conductive polymeric composites undergoes an insulator- conductor transition which depends on the particles' concentration [ 8 , 23]. The threshold value of particles' volume concentration at which these systems become conducting varies in the different systems, e.g. 8\% for carbon black in Araldite D [23], 37\% for $\mathrm{Ag}$ particles in Bakelite powder [24], 5-6\% in systems obtained by compaction of polyethylene powders with $\mathrm{Ni}$ or polyvinyl-chloride with $\mathrm{Cu}[25,26]$, about $40 \%$ for epoxy resin randomly loaded with $\mathrm{Cu}, \mathrm{Ag}, \mathrm{Al}, \mathrm{Sn}$ or $\mathrm{Pb}$ [27] or around 20\% according to other researchers [28].

Complex conductivity and dielectric permittivity as a function of composition and constituents' properties can be calculated using mixing laws [29, 30] and effective medium theories [31].

The aim of this work is to study the effect of concentration of metal particles on the corrosion performance of steel specimens coated with metal filled particulate composites when exposed to saline environment. A commercially available epoxy resin and iron powder were used to prepare 
composites with concentration of iron, 7.5,15 and 30\% wt./wt. Corrosion potential, corrosion current density (Tafel), a.c. impedance

and dielectric measurements were performed.

\section{Experimental}

The test specimens were circular steel disks (diameter $\Phi=6 \mathrm{~cm}$ ) with a composition (in wt.\%) of C, 0.12; Mn, 0.5; P, 0.005; S, 0.05 and balance Fe. The coating used was a commercially available bisphenol A-type epoxy resin (DER 321, Dow Chemical Co.) with epoxide equivalent weight $182-192$, viscosity $500-700 \mathrm{mPa}$ s at 25 ' $\mathrm{C}$ and density $1.14 \mathrm{~g} \mathrm{ml}$ '' at 25 ' $\mathrm{C}$. As curing agent, a cycloaliphatic amine (product XZ 87706.05, Dow Chemical Co.), was used. The ratio resin/curing agent was the stoichiometric according to the manufacturers' data sheets. The system was used either in this formulation or filled with powdered metal in a content of $7.5,15$ or $30 \%$ by weight. Powdered metal was incorporated in the liquid state at $30{ }^{\circ} \mathrm{C}$. Metal powder of iron (Ferak Lab.) of $40 \mathrm{pm}$ grain size was used. The corrosion environment was prepared by dissolving analytical grade $\mathrm{NaCl}$ in deionized water to give $3.5 \mathrm{wt} . \%$.

The metallic substrate was cleaned by immersion in a strong solution of $\mathrm{HCl}$ with an organic corrosion inhibitor, washed with water, polished with emery paper up to 1000 grit, degreased for $10 \mathrm{~min}$ in boiling xylene and stored in a desiccator, containing silica gel, before coating to avoid any influence of moisture. Each mixture after being stirred thoroughly was deposited on the metal surface by a spinning coater (Erichsen 334/E). Initial curing was carried out at ambient for $24 \mathrm{~h}$, followed by postcuring at $40{ }^{\circ} \mathrm{C}$ for $48 \mathrm{~h}$, to assure a stable situation at room temperature. Then the specimens were immersed in the corrosive environment, i.e. in $3.5 \% \mathrm{NaCl}$ solution. Specimens coated only with epoxy

resin were used as reference specimens.

Each type of measurement at every exposure time tested was performed on different specimens and was always duplicated. Specimens having a dry film thickness at each surface equal to $70 \pm 5$ $\mu \mathrm{m}$ were chosen from a greater production number.

The electrochemical cell for both EIS and Tafel measurements consisted of a hollow Teflon ${ }^{\circledR}$ cylinder or pipe clamped to the surface of the metal substrate and creating by an Oring seal the vessel for the electrolyte $(3.5 \% \mathrm{NaCl})$. The working electrode was the coated specimen, having an exposed surface area equal to $19.63 \mathrm{~cm}^{2}$. The reference electrode was a saturated calomel 
electrode (SCE), while the auxiliary electrode was a high density graphite rod. All measurements were carried out at ambient with the electrolyte in contact with air. Before each measurement, the coated steel electrode was kept at open circuit and the change of the potential was recorded until its stabilization at a value referred to as the corrosion potential, $E_{c o r r}$. Potentiodynamic scans were performed, one for the anodic and one for the cathodic polarization, using a Princeton Applied Research model 33 potentiostat/galvanostat. Both anodic and cathodic potentiodynamic scans were run at $1 \mathrm{mV} \mathrm{s}^{-1}$.

Time dependence of the rest potential of each type of specimen referring to a SCE was monitored.

The EIS measurements were performed by applying a small-amplitude perturbation of $15 \mathrm{mV}$ in a sine wave form by means of a Video bridge T-2100 of Electra-Scientific Industries Inc., in the frequency range $20 \mathrm{~Hz}-5 \mathrm{KHz}$ and by an impedance analyser LF4192A of Hewlett Packard, in the frequency range $5 \mathrm{KHz}-13 \mathrm{MHz}$. The modulus of impedance and the phase shift were obtained. Each value was the mean value of five measurements in a logarithmic sweep of the frequencies (20 points per logarithmic unit). Curve fitting of the EIS data was performed by ZSIM software using a non-linear least-squares fitting method (NLLSF) . Dielectric measurements were performed in a three terminal guarded system, constructed according to ASTM D150-92. At predetermined immersion times, coated specimens were taken out of the corrosive environment, washed with acetone, dried in air and were put in the three terminal dielectric cell, so that they formed the dielectric element of the capacitor. Capacitance values and the loss factor (D) of the coating were measured in the frequency range $20 \mathrm{~Hz}-13 \mathrm{MHz}$ using the above-mentioned bridges. From these measurements the dielectric permittivity and dielectric losses were calculated [32].

\section{Results and discussion}

The corrosion tendency of the steel substrate was estimated for each type of coated specimen by the half-cell potential development versus the exposure time to the corrosive environment. Plots of this evaluation referred to a saturated calomel electrode are given in Fig. 1, for specimens coated with epoxy resin or with composites of epoxy resin and iron powder together with that of an uncoated sample used as a reference. 
Immediately after immersion in $3.5 \% \mathrm{NaCl}$ the uncoated specimens exhibit potential values close to $-670 \mathrm{mV}$ and after $4 \mathrm{~h}$ of immersion the potential value of these specimens appears to acquire a constant value of about $-700 \mathrm{mV}$ which remains stable during the whole immersion period.

At the beginning of the exposure, specimens coated with epoxy resin containing iron powder have a potential value close to that of the specimens coated with pure epoxy resin irrespective of the concentration of iron particles. This value is always less electronegative than that of the uncoated specimens. Thereafter a decay to more negative potential values was observed during the first seven days for specimens with coatings containing iron followed by a more or less steady state value of about $-600 \mathrm{mV}$. The potential decay for specimens with pure epoxy coating lasted about 60 days and afterwards a tendency to stabilization was evident at about the same potential region of $-610 \mathrm{mV}$. The corrosion potential provides a qualitative clue whether a given structure is in an active or a passive state. In all cases the trend to reach stable values, which differ less than $100 \mathrm{mV}$ from that of the free corrosion potential of uncoated steel, implies an increasing electrochemical activity of the metal surface during the test.

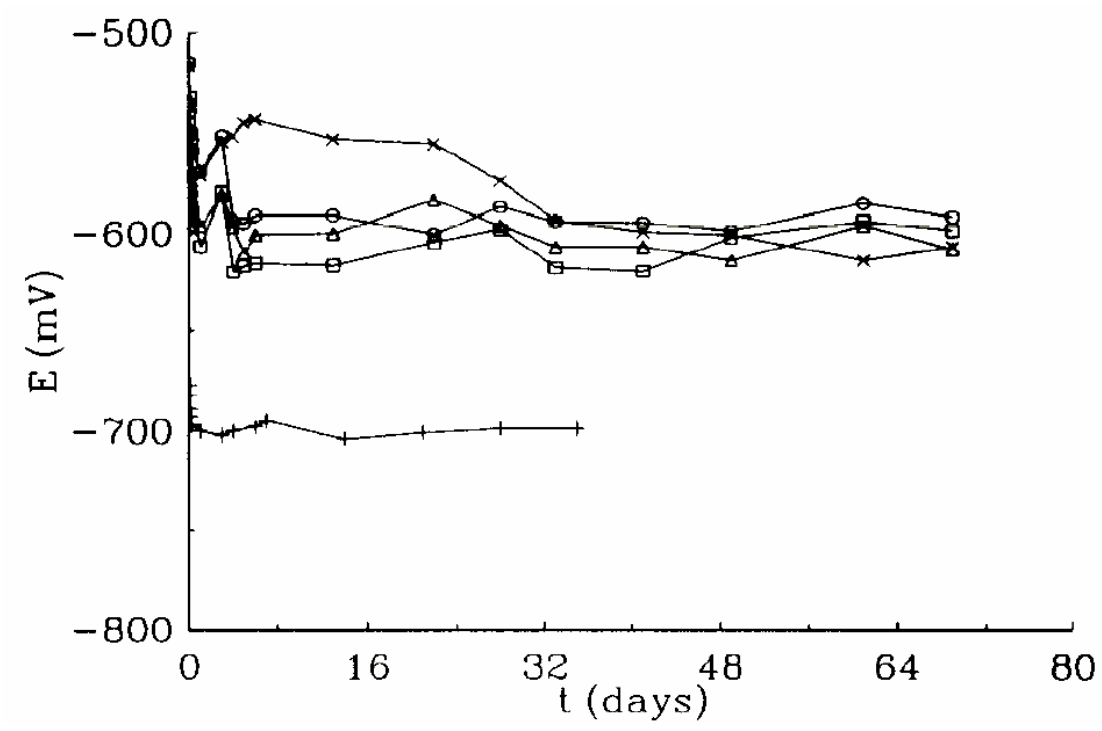

Fig. 1. Time dependence of corrosion potential for different types of coated and uncoated steel specimens: + , bare steel; $\times$, pure epoxy; $\triangle, 7.5 \% ; \bigcirc, 15 \% ; \square, 30 \%$ wt./wt. iron powder. 
However, the evolution of the similar free corrosion potential and small differences in the corrosion potential plateau values irrespective of iron powder concentration do not allow a qualitative prediction of corrosion performance.

The results of anodic and cathodic polarization tests are given in Figs. 2 and 3 for specimens coated with pure epoxy resin or with epoxy resin in combination with iron powder at the beginning and after ninety days of immersion. Each plot is composed of a separate anodic and cathodic scan.

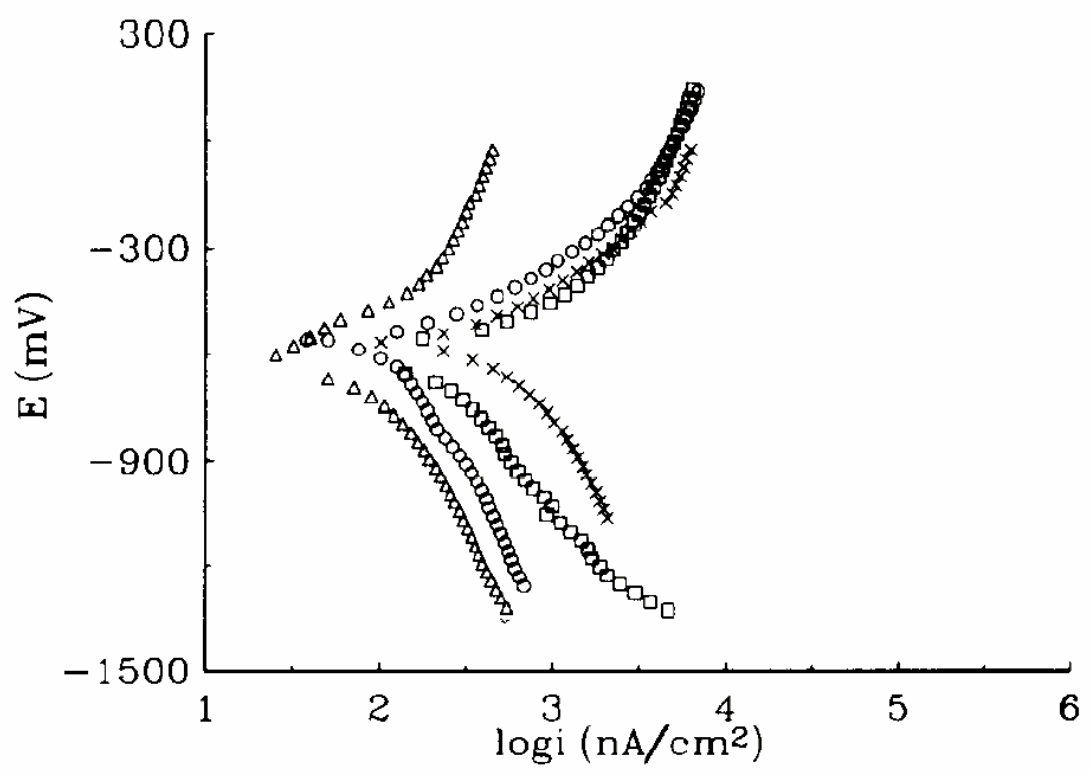

Fig. 2. Potentiodynamic polarization plots of different types of coated steel specimens exposed to $3.5 \% \mathrm{NaCl}$ solution for $4 \mathrm{~h}: X$, pure epoxy; $\triangle, 7.5 \% ; \bigcirc, 15 \% ; \square, 30 \%$ wt./wt. iron powder. 


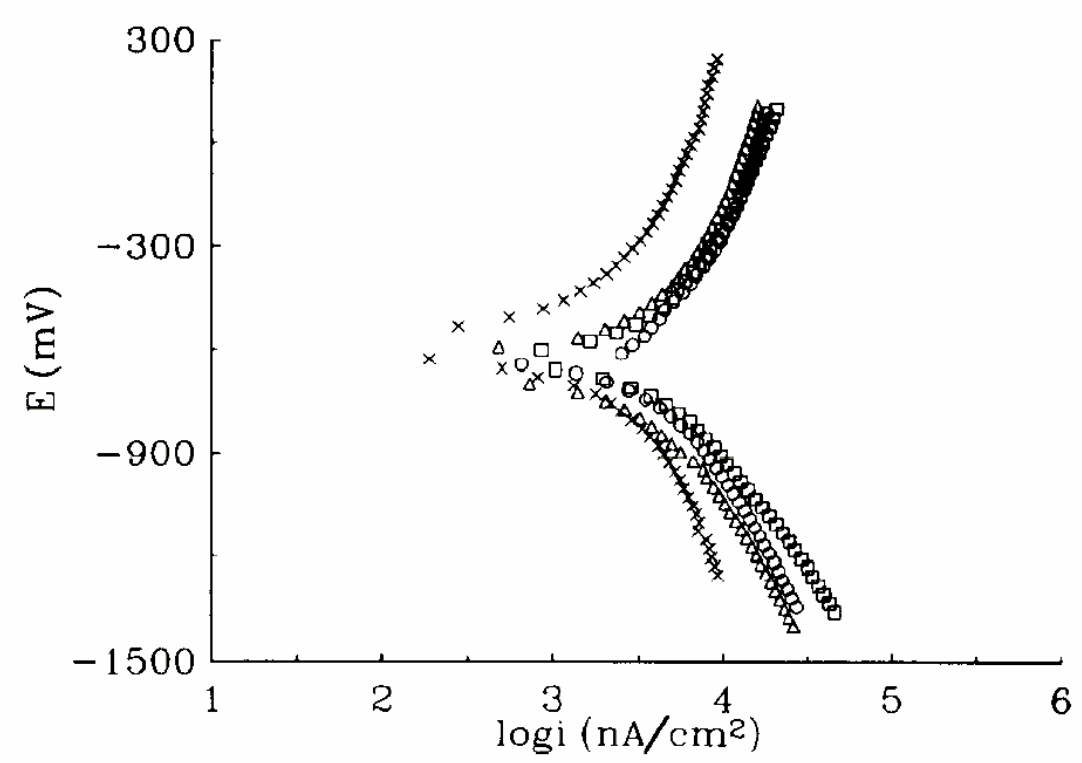

Fig. 3. Potentiodynamic polarization plots for different types of coated steel specimens exposed to $3.5 \% \mathrm{NaCl}$ solution for 90 days: $X$, pure epoxy; $\triangle, 7.5 \%$;, $15 \%$; $\square, 30 \%$ wt./wt. iron powder.

These figures show that the corrosion potential of all tested samples has roughly the same value, an observation which is in agreement with the results of the time dependence of the corrosion potential.

Fig. 2 demonstrates that at the beginning of the exposure, the corrosion current density for all specimens coated with particulate composites is lower than that of specimens coated with pure epoxy resin.

The dispersion of conductive particles in an epoxy matrix changes the transport properties of the coating because it leads to a decrease of the coating resistance and to an easy creation of conductive paths affecting negatively the protective properties of the coating. However, at the same time, the metal powder grains, by filling the micropores of the polymeric matrix, exert a barrier effect which positively affects the protective properties of the coating. Consequently specimens with coating containing iron powder show improved corrosion behaviour.

Coatings with the lowest iron powder content (7.5\% wt.1 wt.) show a clearer improvement than coatings with higher iron powder content $(15,30 \% \mathrm{wt} . / \mathrm{wt}$.), the corrosion performance of which is close to that of the pure epoxy resin. This behaviour is probably the result of a counterbalance 
between the barrier effect exerted by the composite coatings and a possible unsatisfactory wetting of the metal particles by the resin and/or a lower resistance of resin/metal particles adhesion to wet conditions, with increasing metal powder concentration.

It is known [33,34] that in the case of conventional pigments in a coating, if water can accumulate at the pigment/binder interface, or porous particles can be formed, the permeability of the coating increases with the pigment volumeconcentration. This statement can be extended for the case of metal powders when used instead of conventional pigments.

After 90 days of exposure to the corrosive environment the corrosion performance of coatings containing iron powder remains close to that of pure epoxy coatings but the slope of the curves $(d i / d E)$ in the former case is slightly steeper.

Visual examination of the specimens reveals in the early phase of exposure, i.e. at around 15 days, the appearance of green coloured spots which develop to greater black regions with increasing exposure time. The initially appearing spots are attributed to the precipitation of green complexes formed in a chloride environment as intermediate in the formation of oxyhydroxides or iron oxides [35]. Iron oxides probably compose the black products possessing a high adherence to the metal surface [36]. The bulky corrosion products can establish a barrier effect and consequently can counterbalance the increase in permeability due to the presence of iron particles.

The formation of corrosion products in a chloride environment can also deteriorate the protective properties because chloride ions can destroy the oxide layer causing pitting corrosion [37]. The visual examination of the bare steel surface during experiments has not revealed any pitting of the surface.

The minor differences in the performance between the composite coatings tested and the pure epoxy resin coatings strengthen the assumption that the iron concentration levels used in these coatings are lower than the percolation threshold and thus the systems have not yet become purely conductive[8, 23].

The a.c. impedance response of the steel specimens with composite coatings is dependent on immersion time in the sodium chloride environment. Bode plots taken after $4 \mathrm{~h}$ of immersion and 90 days of immersion are displayed in Figs. 4 and 5, respectively. At the frequency range of the performed measurements, $20 \mathrm{~Hz}-13 \mathrm{MHz}$, normally only the response of the organic coating is observed while the response of the corroded substrate is observed in a lower frequency range ( 
$\left.10^{-3}-10 \mathrm{~Hz}\right)$. At the beginning of corrosion this range can be extended and the breakpoint frequency can be shifted from $10^{-3}$ to $10^{4-5}$ or more $\mathrm{Hz}$ [38] but this behaviour cannot be related to any of the specimens tested.

At early exposure times two capacitive responses and consequently two maximum phase shifts are displayed for composite coatings. This implies that in this case two time constants exist [39], representing two rate-determining steps of the electrochemical process, one at high frequencies which is generally attributed to the film and the other at middle frequencies to the presence of iron powder. Coatings of pure epoxy resin show one time constant.

The time dependence of the impedance values (Fig. 6) demonstrates that the coating behaviour passes through a transition state at early immersion times, indicating the change in the properties of the coating, to a steady state in which the impedance values of pure epoxy resin coatings and composite coatings range between 0.4 and $0.7 \times 10^{6} \Omega \mathrm{cm}^{2}$.

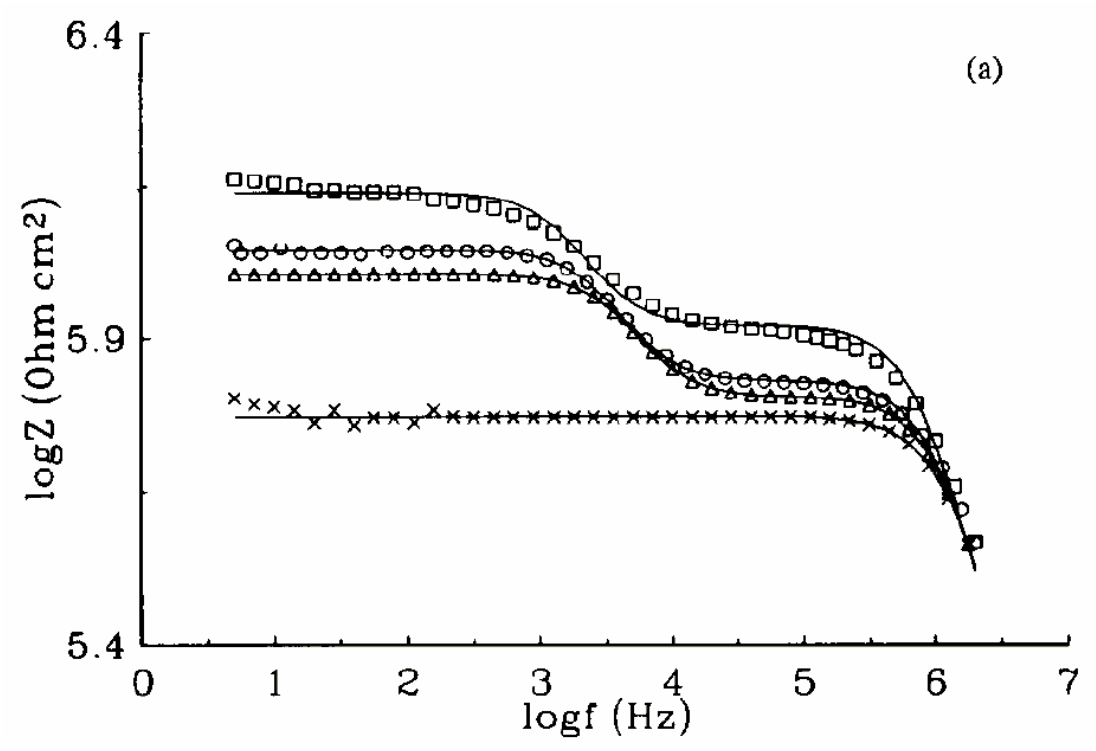




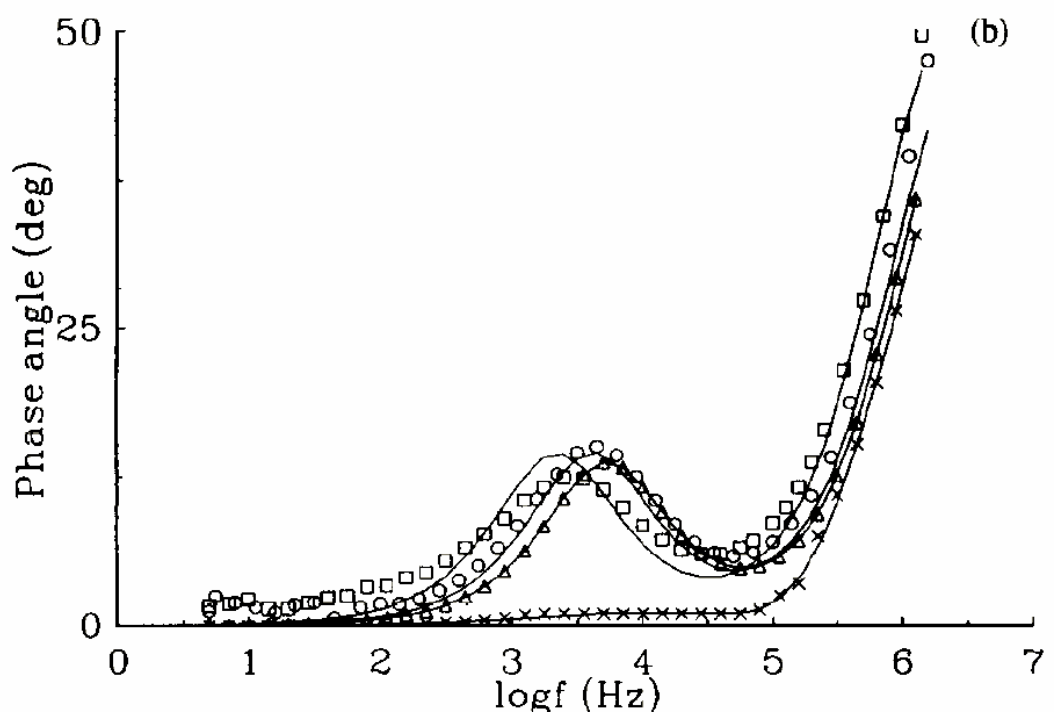

Fig. 4. Bode plots (a) $\log |Z|$ and (b) phase angle, for different types of coated steel specimens exposed to $3.5 \% \mathrm{NaCl}$ solution for $4 \mathrm{~h}$ : $X$, pure epoxy; $\triangle, 7.5 \%$; $\bigcirc, 15 \%$; $\square, 30 \%$ wt./wt. iron powder.

The generally accepted equivalent circuits for barrier and non-barrier type coatings [40-42] are given in Fig. 7. The bulk characteristics of the coating are described by the coating resistance $R_{f}$ and coating geometrical capacitance $C_{f}$. The corrosion process of the substrate is represented by the faradaic impedance $Z$ and the double layer capacitance $C_{d l}$ of the metalcoating interface. $R_{e}$ represents the electrolyte solution resistance. 

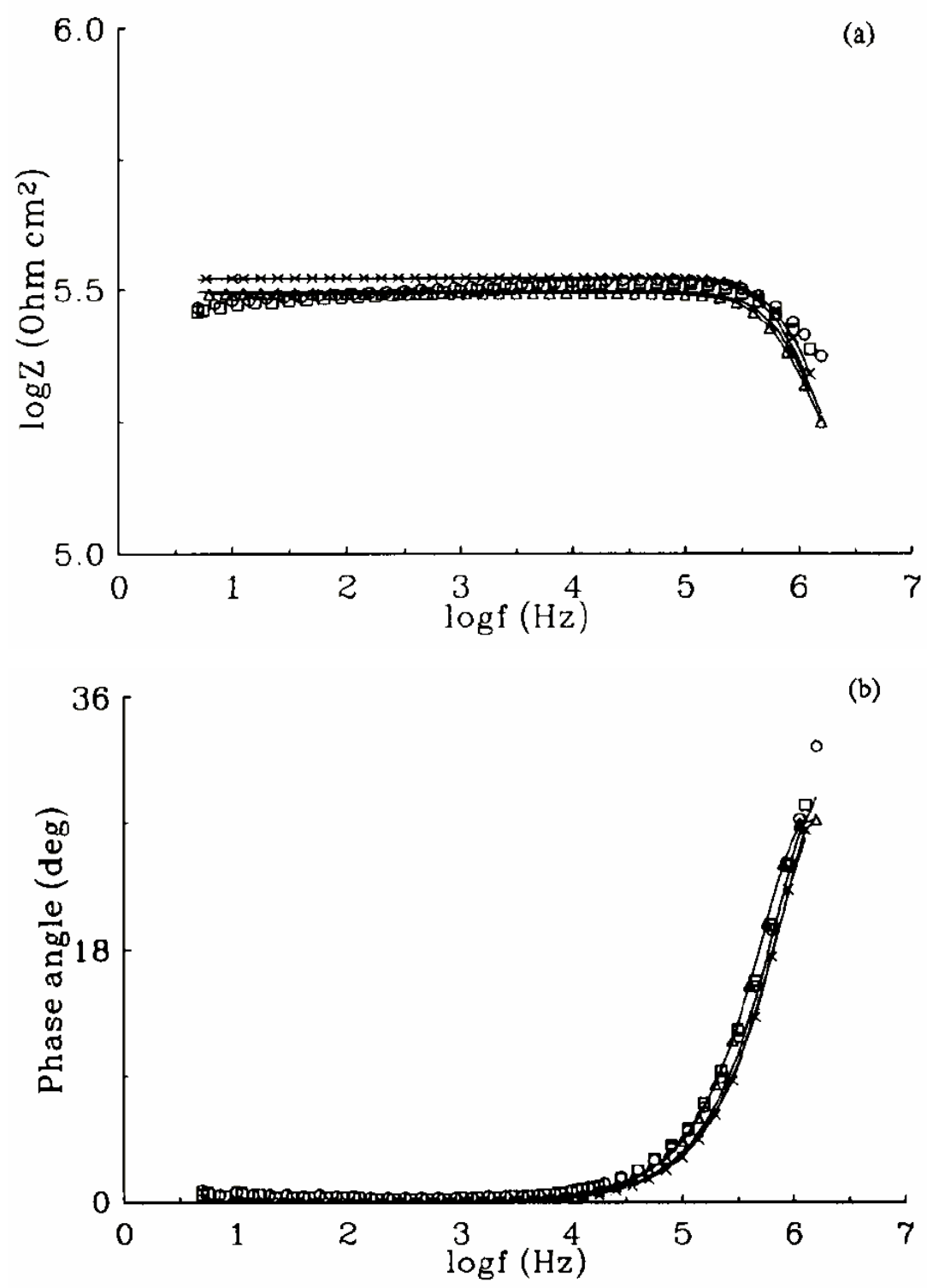

Fig. 5. Bode plots (a) $\log |Z|$ and (b) phase angle, for different types of coated steel specimens exposed to $3.5 \% \mathrm{NaCl}$ solution for 90 days: $X$, pure epoxy; $\triangle, 7.5 \%$; $\bigcirc, 15 \%$; $\square$, 30\% wt./wt. iron powder.

The best fit of the EIS data and the simulated spectra are shown in Figs. 4 and 5 (full lines). The part of the circuit in Fig. 7(b) which generally represents the corrosion process

of the metal substrate was suggested here to express the corrosion process of the iron filler at early immersion times.

If the two time constants of this system are expressed as 
$\tau_{f}=R_{f} C_{f}$ and $\tau_{m c}=R_{m c} C_{m c}$

where $R_{m c}$ and $C_{m c}$ refer to the charge transfer resistance and capacitance of the metal powder corrosion process, the time constant at higher frequencies may be attributed to the coating if $\tau_{f} \leq \tau_{m c}[43]$, which is true in this case.

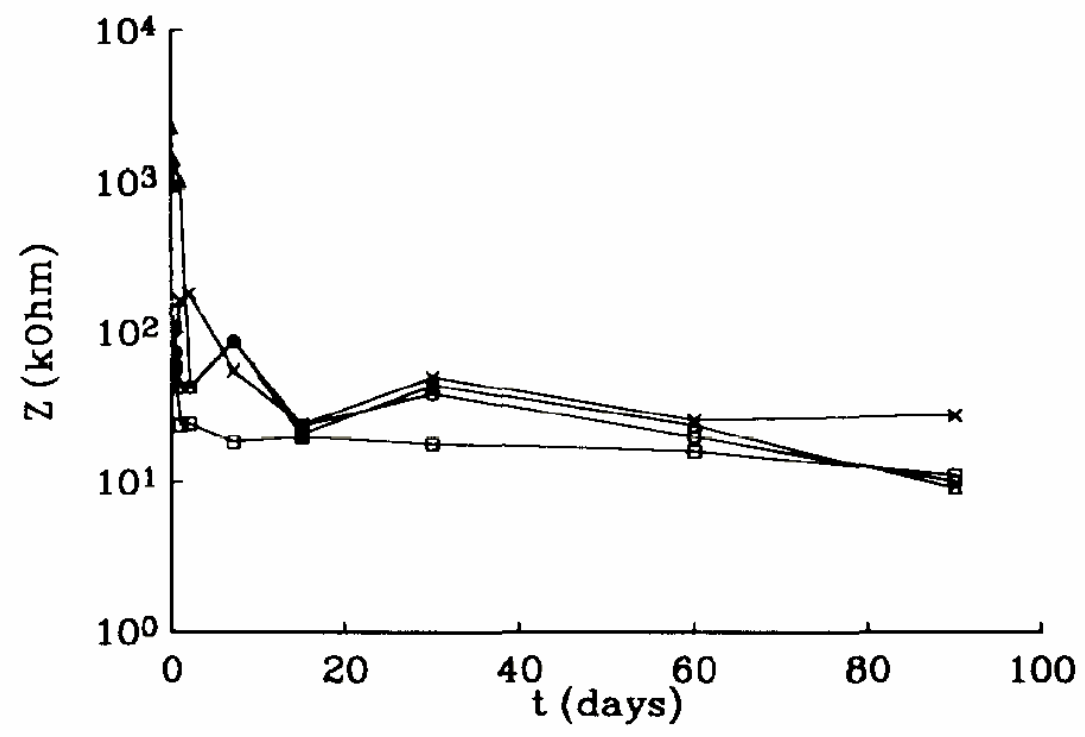

Fig. 6. Impedance time dependence for different types of coated steel specimens: $X$, pure epoxy; $\triangle, 7.5 \% ; \bigcirc, 15 \% ; \square, 30 \%$ wt./wt. iron powder.
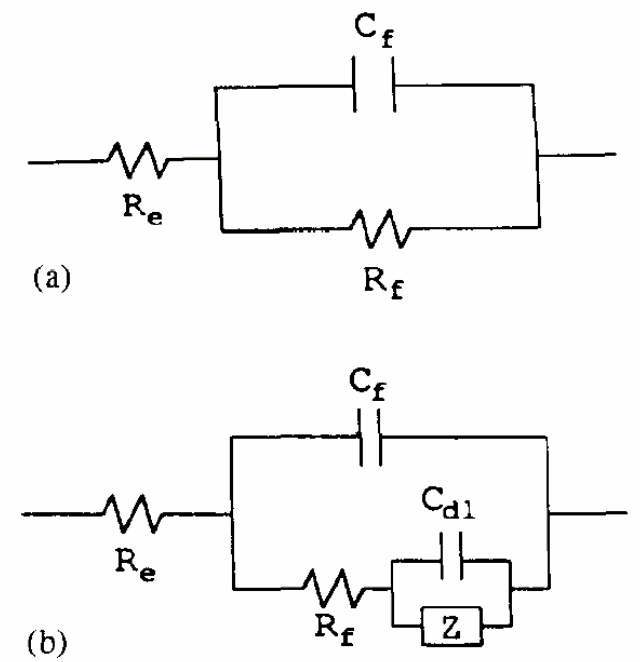

Fig. 7. Electrical equivalent circuit generally accepted for a coated metal/solution interface without diffusion: (a) barrier coatings; (b) non-barrier coating. 
The results show that the equivalent circuit in Fig. 7(b) and in Fig. 7 (a) represent fairly well the electrochemical situation of the composite coatings at early and long immersion times, respectively.

In the region of medium frequencies $\left(\sim 10^{2.5}-10^{4.5} \mathrm{~Hz}\right)$, the time constant is indicative of the oxidation of the metallic grains used as filler, while at high frequencies $\left(>10^{5} \mathrm{~Hz}\right)$ the time constant is attributed to the capacitive behaviour of the polymeric film. The absence of a medium frequency time constant in responses at long immersion times, can be associated with the evolution of corrosion products throughout the film which modifies the characteristics of the coating.

At a high frequency range, resistance exhibits values of about and $1.1 \times 10^{6} \Omega \mathrm{cm}^{2}$. This high value cannot simply represent the electrolyte solution resistance, the value of which is only about $20 \Omega \mathrm{cm}^{2}$ Similar results have been attributed to a contact resistance which imposes a hindrance to movement of ions between electrolyte and coatings [44].

In Fig. 8 the time dependence of the resistance of the composite coatings is reported. In the initial period a sharp reduction of the coating resistance is clearly observed indicating increasing conductivity in the coating and consequently lower protective properties. These results suggest that the penetration of the coating by the electrolyte solution increases rapidly at early immersion times. Thereafter the reduction of the coating resistance slows down and finally at long exposure times a tendency to reach a constant resistance value is evidenced. At the time when a steady state condition is attained, total penetration of the coating by the electrolyte solution occurs which is followed by loss of protection [ 45].

These results reveal that composite coatings with iron concentration up to $30 \%$ behave almost the same as those with pure epoxy resin.

The time evolution of the coating capacitance (Fig. 9) is in accordance with the above results. At early stages of exposure the sharp rise in the capacitance values suggests a rapid water penetration followed by a rather low increase of water diffusion. However, the only information that can be derived concerns the total water in the system, which is higher in the case of composite coatings, and not the amount of aqueous phase water at the metal-coating interface which is the medium where the corrosion of the substrate occurs. 


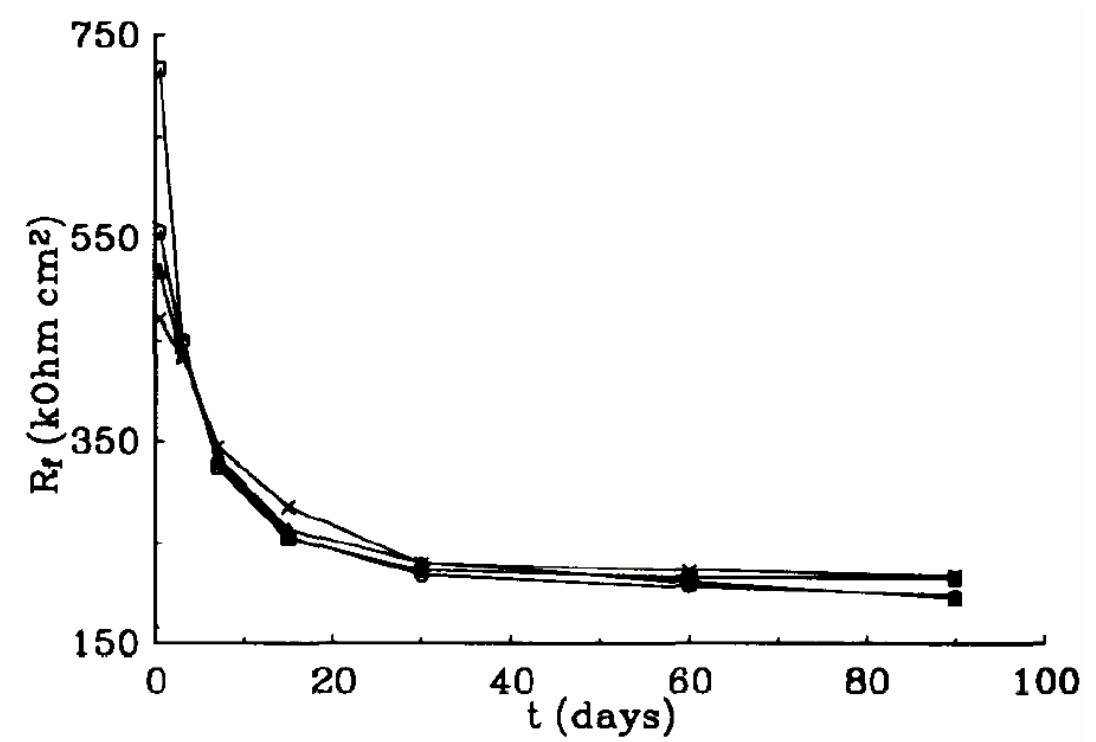

Fig. 8. Change of coating resistance $R_{f}$ with exposure time for different types of coated steel specimens obtained by ZSIM: $\times$, pure epoxy; $\triangle, 7.5 \% ; \bigcirc, 15 \%$; $\square, 30 \%$ wt./wt. iron powder.

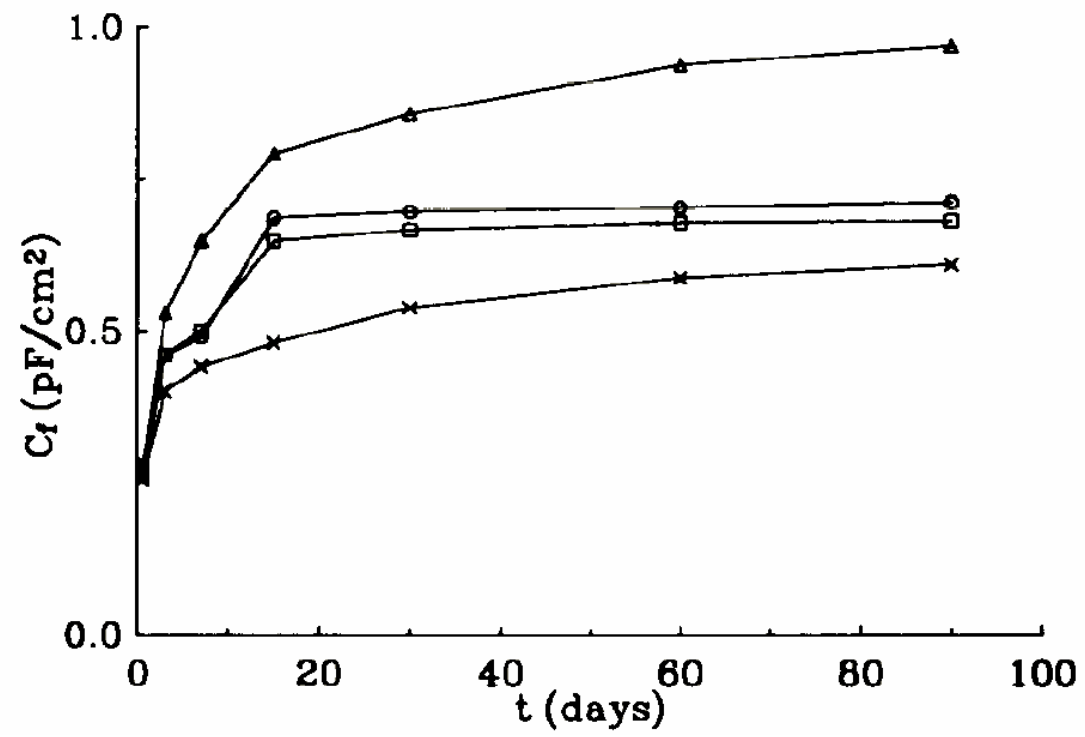

Fig. 9. Change of coating capacitance $\mathrm{C}$, with exposure time for different types of coated steel specimens obtained by ZSIM: $X$, pure epoxy; $\triangle, 7.5 \% ; \bigcirc, 15 \%$; $\square, 30 \%$ wt./wt. iron powder. 


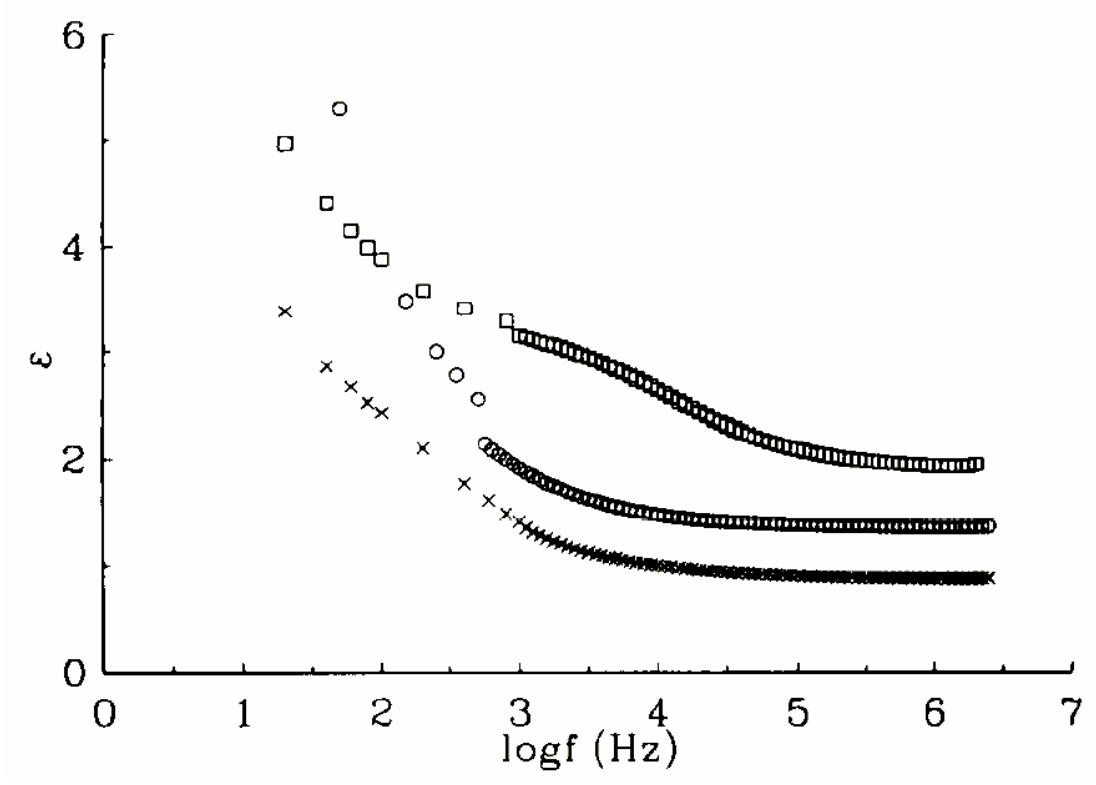

Fig. 10. Dielectric permittivity vs. frequency for different types of coated steel specimens after one day of immersion: $X$, pure epoxy; $\bigcirc, 15 \%$; $\square, 30 \% \mathrm{wt} . / \mathrm{wt}$. iron powder.

The dielectric response of the coatings can also be related to their corrosion behaviour. Fig. 10 shows the dielectric permittivity versus frequency, of the coatings containing various amounts of iron powder, after one day of immersion in the corrosive environment.

As expected dielectric permittivity is higher for the coatings containing iron powder than for those with pure epoxy resin. Dielectric permittivity also increases with the amount of iron powder in the coatings. In the low frequency range dielectric permittivity shows high values which are diminished as the frequency is increased, a behaviour which can be attributed to the interfacial polarization effect. This phenomenon appears in heterogeneous media consisting of phases with different dielectric permittivities and conductivities and is due to the accumulation of charges at the interfaces [46-48].

There are many causes for heterogeneity in materials but those concerning the coatings used in the present work relate to interfaces created by particles dispersed in the insulating matrix, by penetrating water and the corrosion products created in the coating.

When an electric field acts on materials it may induce polarization provided that there are charges or dipoles which are capable of being spatially redistributed under the field. For dipolar polarization charge redistribution depends on dipolar re-orientation whilst for electronic and atomic polarizations charge displacements occur at optical frequencies. Interfacial polarization, however, can involve several alternatives, including migration of charges through the bulk of the 
materials, surface conductance or transport within any double layer. It can thus achieve large magnitudes (reflected in high permittivity) because of the large distances, in terms of molecular scale, that carriers can be displaced. Even a very small number of carriers can provide large induced dipoles across the regions of heterogeneity.

As the frequency of the acting electric field is increasing, orientation of the large dipoles becomes more difficult and consequently the dielectric permittivity is decreased.

After 60 days of immersion the situation has become more complicated (Fig. 11). The water uptake leads to the transformation of the surface of the metal particles and the creation of corrosion products. Values of dielectric permittivity are higher than the values after one day of immersion, mainly because of the higher content of water in the composite coatings. The coatings with $7.5 \% \mathrm{wt}$./wt. iron content show higher values of dielectric permittivity than the coatings with $15 \%$ or $30 \%$ wtlwt. in iron powder.

In the coating with the lowest amount of iron particles, the modification of the characteristics of the coating by the oxidation of the metallic grains and the creation of corrosion products is also low and does not significantly influence the permittivity. On the contrary when the amount of iron particles in the coating is high, corrosion products, which generally have low dielectric permittivity, will be present in greater amounts and according to the law of mixtures, the dielectric permittivity will be lower for the coatings with higher initial loading in iron particles.

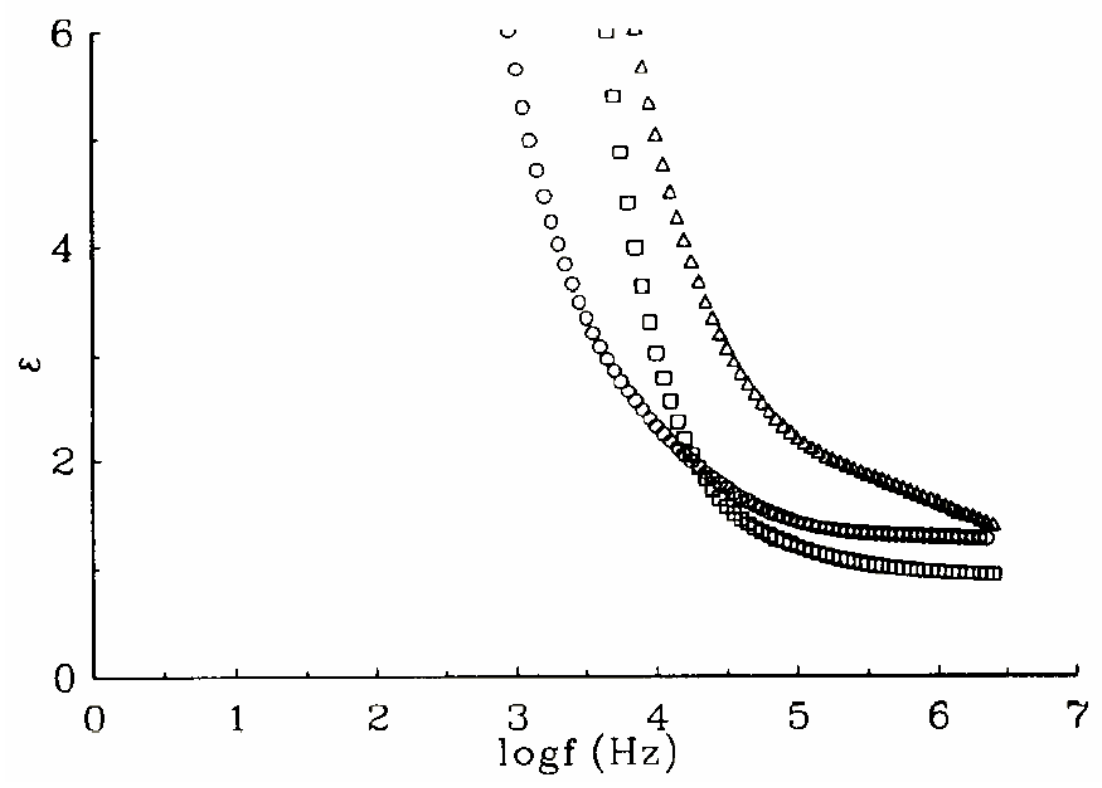


Fig. 11. Dielectric permittivity vs. frequency for different types of coated steel specimens after 60 days of immersion: $\triangle, 7.5 \% ; \bigcirc, 15 \% ; \square, 30 \%$ wt./wt. iron powder.

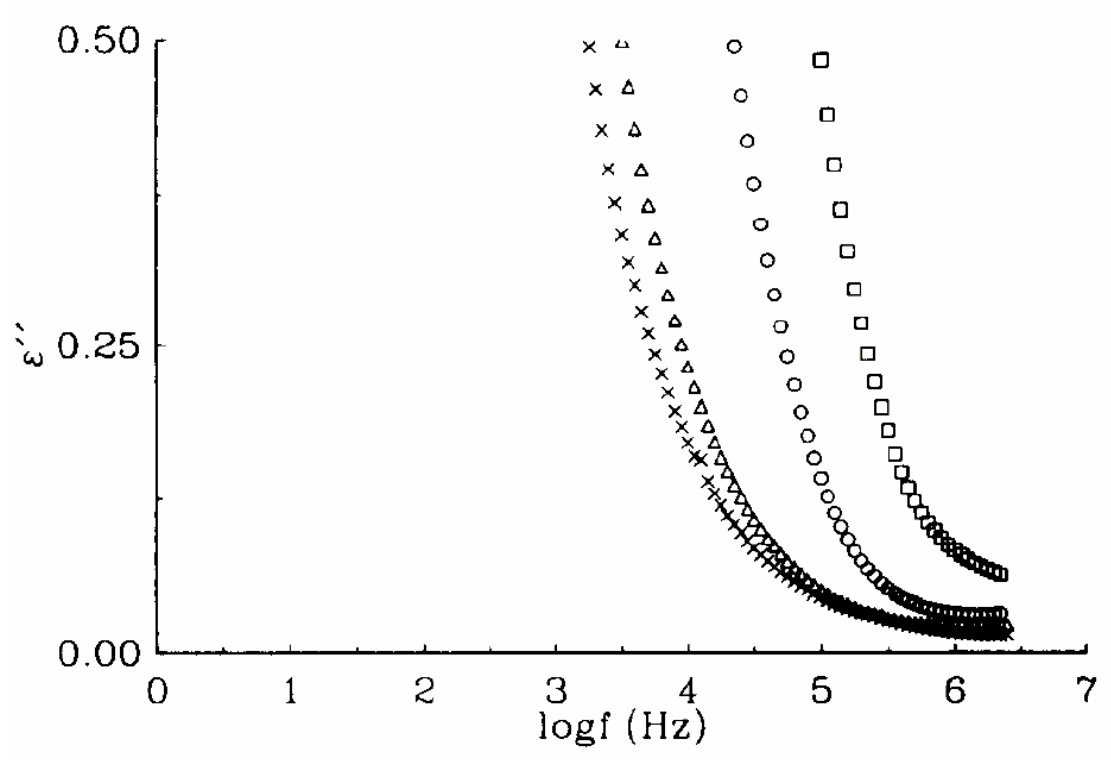

Fig. 12. Dielectric loss vs. frequency for the coating with $15 \% \mathrm{wt} . / \mathrm{wt}$. iron powder for different times of immersion to the corrosive environment: $\triangle, 1 ; \bigcirc, 15 ; \square, 60$ days. The pure epoxy coating after 1 day of immersion is represented by $X$.

The dielectric losses versus frequency of the coating with pure epoxy after one day of immersion in the corrosive environment and of the coating with $15 \% \mathrm{wt} . / \mathrm{wt}$. iron powder for various immersion times are shown in Fig. 12. All coatings show considerable losses in the low and middle range of frequencies as expected because of interfacial polarization. As immersion time increases and water uptake is increased in the coatings dielectric losses increase and move to higher frequencies.

The high value of the slope in curves at low frequencies reveals the existence of conductive pathways [49] but since the slope is not substantially altered as immersion timeincreases, the conductivity in the coating is not considerably changing, which means that the protective behaviour still exists. Almost the same behaviour was observed for all coatings irrespective of the loading in metal powder. Small differences in the values of the dielectric losses of coatings with different iron content do not reflect any real change in their behaviour when exposed to a corrosive environment. Almost the same slope of the loss curve representing the losses of the pure epoxy coating compared with those of coatings containing iron powder also ascertains what 
was already found by other measurements: iron in the coating, at least, does not deteriorate the protective properties of the coating.

\section{Conclusions}

Steel specimens coated with particulate polymeric coatings composed of epoxy resin and iron powder in various concentrations show an increasing electrochemical activity of the metal surface during exposure to $3.5 \% \mathrm{NaCl}$ solution. This development forwards an active state similar to that of specimens coated with pure epoxy resin.

Corrosion rate measurements of the steel substrate reveal minor differences in the anticorrosive behaviour of the composite coatings, irrespective of the iron content in the coating, their action being always close to that of pure epoxy resin coatings.

These results are in good correlation with the corresponding EIS measurements, according to which the resistance of both the composite and the pure epoxy resin coatings is rapidly diminished at early exposure times but thereafter it shows a tendency to a rather steady state situation, while the coatings' capacitance first shows a sharp rise followed by a very low increase. It is suggested that the evolution of corrosion products throughout the film modified the characteristics of the coating exerting a barrier effect, counterbalancing the increase in conductivity. The negative effect of the presence of chloride ions on the protective properties of the coating due to the formation of very soluble iron salts (halogenides) which destroy the oxide layer and cause pitting corrosion has not been observed.

The dielectric approach to the corrosion behaviour of the coatings reveals also the formation of corrosion products which decrease the dielectric permittivity of the coatings with higher metal content, at long exposure times. Dielectric losses, reflecting the change in conductivity of the coatings with time and metal loading, signify that these polymeric particulate composite coatings present a similar anticorrosive ebehaviour to the coatings of pure epoxy resin.

\section{References}

[1] P.A. Scheweitzer, Corrosion and Corrosion Protection Handbook, Marcel Dekker, New York, 1983, p. 375.

[2] M.G. Fontana and N.D. Greene, Corrosion Engineering, McGraw- Hill, New York, 2nd edn., 1978, p. 188. 
[3] B. Ellis, Chemistry and Technology of Epoxy Resins, Blackie, London, 1993, p. 216.

[4] B. Normand, A. Pierre and J. Pagetti, in J.M. Costa and A.D. Mercer (eds.), Progress in the Understanding and Prevention of Corrosion, Vol. 1, Institute of Materials, London, 1993, p. 149.

[5] Z. Klenowiczand A. Miszczyk, in J.M. Costaand A.D. Mercer (eds.), Progress in the Understanding and Prevention of Corrosion, Vol. 1, Institute of Materials, London, 1993, p. 222. [6] M.M. Schwartz, Composite Materials Handbook, McGraw-Hill, New York, 1984, p. 376.

[7] 1. Delmonte, Metal/Polymer Composites, Van Nostrand Reinhold, New York, 1990, p. 163 and 171.

[8] C. Pierre, R. Deltour, J.A.A.J. Peterboom and P.J.M. Van Bentum, Phys. Rev. B, 42 (6) ( 1990) 3380 .

[9] D.E. Davenport, Polym.-Plast. Technol. Eng., 17 (1981) 211. [10] P.S. Neelakanta, Electron Packag. Prod., 29 ( 1989) 64.

[11] R.J. Frame and D.J. Tedford, IEEE Trans. Electr. fnsul., ET-21 ( 1986) 23.

[12] P.S. Theocharis, S.A. Paipetis and G.S. Papanicolaou, J. Appl. Polym. Sci., 22 (1978) 2245.

[13] M. Oda. Y. Shibara, T. Yamamotoand T. Morita, in M.W. Kendigand J. Leidheiser (eds.), Corrosion Protection by Organic Coatings, Electrochemical Society, NJ, 1987, p. 262.

[14] A. Al-Hashem and D. Thomas, J. Coat. Technol., 62 (783) (1990) 51.

[15] D.J. Frydrych, G.C. Fart-\&ton and H.E. Townsend, in M.W. Kendig and J. Leidheiser (eds.), Corrosion Protection by Organic Coatings, Electrochemical Society, NJ, 1987, p, 240.

[16] M. Kendig, S. Jeanjaquet, J. White and F. Mansfeld, in M.W. Kendig and J. Leidheiser (eds.), Corrosion Prorecrion by Organic Coatings. Electrochemical Society, NJ, 1987, p. 253.

[17] N. Kouloumbi, G.M. Tsangaris, A. Skordos, P. Karkanas and K. Kyriopoulou, Mater. Sci. Forum, 192-194 (1995) (Part2) 813.

[18] N. Kouloumbi, G.M. Tsangaris and ST. Kyvelidis, J. Coat Technol., 66(839) (1994) 83.

[19] R.P. Sheldom, Composite Polymeric Materials, Applied Science, London, 1982, p. 110.

[20] M. Stratmann and K. Hoffmann, Proc. 9th European Gong. Corrosion, Ukecht, Netherlands, 1989, Vol. II, Paper CO-070.

[21] C.M. Hendry, J. Coat. Technol. 62 (786) (1990) 33.

[22] W. Bosch and W. Funke, Proc. XXth FATIPEC Congr., Nice, France, 1990.

[23] J.L. Miane, M.E. Achour and F. Carmona, Phys. Status Solidi A, 1 (1984) K71.

[24] J. Gurland. Trans. Metall. Sot. AIME, 236 ( 1966) 642. 
[25] A. Malliaris and D.T. Turner, J. Appl. Phys., 42 (1971) 614.

[26] J. Swapan and K. Bhattacharya, Polymer, 20 ( 1979) 116.

[27] F.F.T. de Aranjo and H.M. Rosenberg, J. Phys. D, 9 (1976) 1025.

[28] S.M. Aharoni, J. Appl. Phys., 43 (1972) 2463.

[29] J. Brown, Prog. Dielecrr.. 2 (1960) 69.

[30] L.K.H. Van Beek, Prog. Dieleclr., 7 (1967) 69.

[31] B.E. Springett, Phys. Rev. Len., 31 (1973) 1463.

[32] G.M. Tsangaris, G.C. Psarras and A.J. Kontopoulos, J. Non-Cryst. Solids, 131-133 (1991) 1164.

[33] F.M. Geenen, Ph.D. Thesis, Delft University of Technology, Netherlands, 1990.

[34] S. Paul, Su\$ace Coafings; Science and Technology, Wiley, New York, 1985, p. 463.

[35] T. Misawa, K. Hashimoto and S. Shimodsiro, Corros. Sci., 14 (1976) 131.

[36] H. Leidheiser, Jr. and M.W. Kendig, Corros. NACE, 32 (2) (1976) 69.

[37] D. Williams, C. Westcott and M. Fleischmann, J. Elecrrochem. Sot., I32 (8) (1985) 1796, 1804.

[38] J.R. Scully, J. Electrochem. Sac., 236 (4) (1989) 979.

[39] J.N. Murray and H.P. Hack, Corrosion, 47 (6) (1991) 480.

[40] F. Mansfeld, Corrosion, 37 (1981) 301.

[41] I F. Mansfeld, M.W. Kendig and S. Tai, Corros. NACE, 38 (9) (1982) 478.

[42] R. BabiC and M. MetikoS-HukoviC, Prog. Org. Coat, 23 ( 1994) 275.

[43] T. Monetta, F. Bellucci, L. Nicodemo and L. Nicolais, Prog. Org. Coat.. 21 (1993) 353.

[44] G.C. Lunazzi and M. Maja, Pirture e Vernici, 60 (7) (1984) 55.

[45] A. Amurudin and D. Thierry, Er. Corros. J., 26 (3) (1991) 195.

[46] R.W. Sillars, J. Inst. Electr. Eng., 80 (1937) 378.

[47] R. Bartnikas, in Bamikas/Eichhom (eds.), Engineering Dielectrics, Vol. IIA, ASTM STP 783, Philadelphia, PA, 1983, p. 61.

[48] P. Hedvig, Dielectric Spectroscopy of Polymers, Adam Hilger, Bristol, 1977, p. 282.

[49] H. Leidheiser, Jr., Corros. NACE, 39 (5) (1983) 189. 